\title{
Intervenções de enfermagem prescritas para pacientes adultos internados em unidade de terapia intensiva
}

Nursing interventions prescribed for adult patients admitted to intensive care unit

Intervenciones de enfermería prescriptas para pacientes adultos internados en unidad de terapia intensiva

\section{Kalliny Nathiara de Oliveira Stralhoti ${ }^{I}$, Fabiana Gonçalves de Oliveira Azevedo Matos ${ }^{\text {II }}$ Débora Cristina Ignácio Alves ${ }^{\mathrm{III}}$, João Lucas Campos de Oliveira ${ }^{\mathrm{IV}}$, Djulia Camila Berwangerv $^{\text {, }}$ Drieli Wawzeniak de Anchieta ${ }^{\mathrm{VI}}$}

\begin{abstract}
Resumo: Objetivo: identificar as intervenções de enfermagem prescritas para pacientes adultos internados em Unidade de Terapia Intensiva. Método: estudo documental, transversal, do tipo mapeamento cruzado, realizado em hospital público de ensino. Foram avaliados 29 prontuários que continham registros de prescrições de enfermagem realizados na admissão do paciente na referida unidade. As intervenções prescritas $(n=725)$ foram mapeadas de acordo com a Nursing Interventions Classification e analisadas por estatística descritiva. Resultados: as 725 intervenções prescritas estavam contidas em 18 "intervenções de enfermagem", nove "classes" e três "domínios". As intervenções "Cuidados da Pele: tratamentos tópicos", "Controle da Pressão", "Supervisão" e "Controle de Infecção"; as classes "Controle de Pele/Feridas" e "Facilitação do Autocuidado" e os domínios "Fisiológico Complexo" e "Fisiológico Básico" foram os mais frequentes. Conclusões: as prescrições dos enfermeiros intensivistas relacionavam-se à promoção e manutenção das necessidades humanas fisiológicas e de segurança.

Descritores: Enfermagem; Processo de enfermagem; Cuidados críticos; Classificação; Unidades de terapia intensiva
\end{abstract}

\footnotetext{
IEnfermeira. Especialista em enfermagem em urgência e emergência do Hospital Pequeno Príncipe. Curitiba, PR, Brasil. Email: kalliny_stralhoti@hotmail.com. Orcid: https://orcid.org/0000-0002-9457-3082.

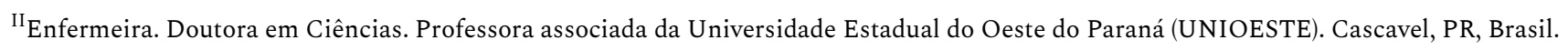
Email: fabianamatos@hotmail.com. Orcid: https://orcid.org/0000-0002-5283-5363.

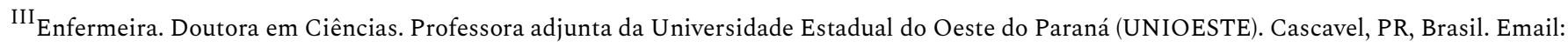
dcialves@gmail.com. Orcid: https://orcid.org/0000-0001-6892-366X.

${ }^{\mathrm{IV}}$ Enfermeiro. Doutor em Enfermagem. Professor adjunto do Departamento de Assistência e Orientação Profissional, Escola de Enfermagem, Universidade Federal do Rio Grande do Sul (UFRGS). Porto Alegre, RS, Brasil. E-mail: enfjoaolcampos@yahoo.com.br. Orcid: https://orcid.org/0000-0002-1822-2360.

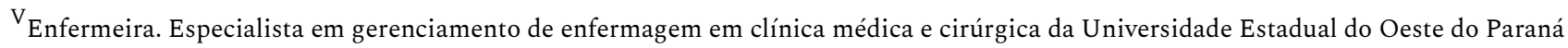
(UNIOESTE). Cascavel, Paraná, Brasil. Email: djuliaberwanger@hotmail.com. Orcid: https://orcid.org/0000-0003-1169-2746.

${ }^{\mathrm{VI}}$ Enfermeira. Especialista em vigilância em saúde e controle de infecção da Universidade Estadual do Oeste do Paraná (UNIOESTE). Cascavel, Paraná, Brasil. Email: drieli_wa@hotmail.com. Orcid: https://orcid.org/0000-0002-9196-1688.
} 
ntervenções de enfermagem prescritas para pacientes adultos internados em unidade de terapia... $\mid 2$

Abstract: Aim: to identify the nursing interventions prescribed for adult patients hospitalized in the Intensive Care Unit. Method: documentary, cross-sectional mapping study conducted at a public teaching hospital. We evaluated 29 records that with nursing prescriptions performed at the patient's admission to the unit. The prescribed interventions $(n=725)$ were mapped according to Nursing Interventions Classification and analyzed using descriptive statistics. Results: The 725 prescribed interventions were in 18 "nursing interventions", nine "classes" and three "domains". The interventions "Skin Care: topical treatments", "Pressure Control", "Supervision" and "Infection Control"; the classes "Skin Control / Wounds" and "Facilitation of Self Care" and "Complex Physiological" and "Basic Physiological" domains were the most frequent. Conclusions: the prescriptions made by intensive care nurses were related to the promotion and maintenance of human physiological and safety needs.

Descriptors: Nursing; Nursing Process; Critical Care; Classification; Intensive Care Units

Resumen: Objetivo: identificar las intervenciones de enfermería prescritas para pacientes adultos internados en Unidad de Terapia Intensiva. Método: estudio documental, transversal, del tipo mapeo cruzado, realizado en un hospital público de enseñanza. Fueron evaluados 29 prontuarios con registros de prescripciones de enfermería realizados en la admisión del paciente en la referida unidad. Las intervenciones prescritas $(\mathrm{n}=725)$ fueron mapeadas de acuerdo con la Nursing Interventions Classification y analizadas por estadística descriptiva. Resultados: las 725 intervenciones prescritas estaban contenidas en 18 "intervenciones de enfermería", nueve "clases" y tres "dominios". Las intervenciones "Cuidado de la Piel: tratamientos tópicos", "Control de la Presión", "Supervisión" y "Control de Infección"; las clases "Control de Piel/Heridas" y "Facilitación del Autocuidado" y los dominios "Fisiológico Complejo" y "Fisiológico Básico" fueron los más frecuentes. Conclusiones: las prescripciones de los enfermeros intensivos se relacionan con la promoción y el mantenimiento de las necesidades humanas fisiológicas y de seguridad.

Descriptores: Enfermería; Proceso de enfermería; Cuidados críticos; Clasificación; Unidades de cuidados intensivos

\section{Introdução}

A enfermagem segue em busca de consolidação e valorização profissional na tentativa de evitar que as suas funções fiquem subordinadas a outras categorias profissionais, e para isso, se esforça para que a assistência de enfermagem seja realizada de forma metodológica, organizada e reflexiva, com o intuito de solidificar o enfermeiro como gestor do cuidado. ${ }^{1}$ Neste contexto, o Processo de Enfermagem (PE) se destaca como um método eficiente para nortear a prática do enfermeiro de forma sistematizada. ${ }^{2}$ 
No Brasil, a Resolução n⿳o 358/2009 do Conselho Federal de Enfermagem (COFEN) determina que o cuidado sistematizado seja realizado em todos os locais onde ocorra o exercício da enfermagem, público ou privado, e cita as cinco etapas que compõem o PE, a saber: investigação ou histórico de enfermagem; diagnóstico de enfermagem; planejamento do cuidado; implementação da assistência de enfermagem; e avaliação. ${ }^{3}$

A primeira etapa consiste no levantamento do histórico de enfermagem. Trata-se do momento em que são investigadas as informações do paciente, da família e do espaço em que o indivíduo vive para que possa ser traçado o perfil do cliente. Na segunda etapa ocorre a identificação e a rotulação dos diagnósticos de enfermagem, por meio da análise cautelosa das informações coletadas. A terceira etapa contempla o planejamento da assistência de enfermagem a ser prestada com foco na obtenção dos resultados esperados que serão discutidos com o paciente e com a família com o intuito de reparar e/ou atenuar os problemas de saúde. ${ }^{2}$

A implementação da assistência de enfermagem acontece na quarta etapa do PE, em que são colocadas em prática as ações anteriormente estabelecidas, portanto, prescritas. Por fim, a quinta e última etapa do PE consiste na avaliação. Nessa fase, serão feitas as ponderações sobre as intervenções realizadas junto ao paciente e serão feitos os registros com relação à resposta ao plano de cuidado prescrito. ${ }^{2}$

O cuidado de enfermagem prestado nos moldes do PE requer embasamento científico e sua utilização fornece ao enfermeiro meios para a gestão do cuidado, proporcionando reconhecimento profissional e conferindo saber próprio/identitário à enfermagem. ${ }^{1,4-5}$

A prescrição de cuidados de enfermagem é ato privativo do enfermeiro. ${ }^{3}$ No entanto, é importante que a equipe de enfermagem esteja engajada nesse processo, uma vez que a execução das ações de cuidado prescritas pelo enfermeiro é tarefa de toda equipe de enfermagem. ${ }^{6}$ 
Intervenções de enfermagem prescritas para pacientes adultos internados em unidade de terapia...l 4

A Unidade de Terapia Intensiva (UTI) é designada para atender pacientes críticos que exigem cuidados complexos, especializados e ininterruptos, e dessa forma, a equipe de saúde que atua nesse setor deve possuir habilidades técnicas conhecimento científico. ${ }^{7}$ Conhecer as particularidades e as especificidades dos pacientes internados em UTI, bem como as suas necessidades de cuidado, contribui para direcionar o planejamento da assistência e promover prescrições de enfermagem adequadas às necessidades do paciente crítico. ${ }^{6}$ Assim, questionouse: Quais são as intervenções de enfermagem prescritas para pacientes críticos? Com isso, o objetivo do estudo consistiu em identificar as intervenções de enfermagem prescritas para pacientes adultos internados em unidade de terapia intensiva.

\section{Método}

Trata-se de um estudo transversal, documental, quantitativo, do tipo mapeamento cruzado. A pesquisa foi realizada na UTI para pacientes adultos de um hospital público de ensino localizado na região oeste do Paraná, Brasil. A referida instituição consistia em um hospital geral e contava com 215 leitos exclusivos à demanda do Sistema Único de Saúde (SUS). Por sua vez, a UTI possuía 14 leitos e a equipe de enfermagem era composta por um enfermeiro coordenador, cinco enfermeiros assistenciais e 21 técnicos de enfermagem. O setor contava também com uma equipe permanente de médicos intensivistas e fisioterapeutas e com serviços especializados de fonoaudiologia, nutrição e odontologia.

Importante destacar que a referida unidade dispunha de sistema computadorizado para o registro dos diagnósticos de enfermagem (DE) identificados e das ações de enfermagem prescritas pelos enfermeiros. Os DE eram documentados de acordo com a classificação de enfermagem padronizada da North American Nursing Diagnosis Association International (NANDA-I), já as atividades de enfermagem prescritas pelos enfermeiros eram registradas de forma livre, sem seguir nenhuma classificação de enfermagem padronizada. 
5 | Stralhoti KNO, Matos FGOA, Alves DCI, Oliveira JLC, Berwanger DCI, Anchieta DW

A pesquisa foi desenvolvida em três etapas. Na primeira etapa, foi verificado o interesse dos enfermeiros prescritores em fazer parte do estudo mediante a assinatura do Termo de Consentimento Livre e Esclarecido (TCLE). Na segunda etapa, foi realizada a seleção dos prontuários que atendiam os critérios de elegibilidade no estudo (ser de paciente com idade igual ou superior a 18 anos, ter registro de prescrição de enfermagem realizada durante a admissão do paciente na UTI e ter TCLE assinado pelo enfermeiro prescritor). Por fim, na terceira etapa foi realizada a coleta de dados propriamente dita, durante 30 dias consecutivos entre junho e julho de 2015.

$\mathrm{Na}$ coleta de dados, uma listagem de pacientes internados na UTI em estudo era consultada diariamente no recorte temporal estabelecido. Havendo a identificação de novo internamento, era procedida a extração dos dados de interesse do prontuário do paciente. Para isso, os pesquisadores elaboraram uma planilha eletrônica que foi impressa para realizar a coleta manual das atividades de enfermagem prescritas pelos enfermeiros, e, posterior mapeamento cruzado com a classificação da Nursing Interventions Classification(NIC). ${ }^{8}$

A NIC é uma classificação que padroniza as intervenções de enfermagem e lista uma série de atividades específicas da profissão, sejam elas independentes ou interdependentes, abrangendo todas as especialidades da enfermagem, podendo ser utilizada nos mais variados campos de atuação (na assistência primária, terciária, domiciliar, escolar, entre outras.). É estruturada em três níveis, sendo constituída por sete domínios, 30 classes e 554 intervenções, totalizando em torno de 13.000 atividades de enfermagem padronizadas. ${ }^{8}$ Cada intervenção de enfermagem classificada possui um conceito, uma lista de possíveis atividades a serem realizadas e as bibliografias de referência. Dessa forma, a NIC oferece subsídios teóricos para apoiar a tomada de decisão tanto de enfermeiros experientes como de alunos ou enfermeiros que estão iniciando a carreira profissional. 
Intervenções de enfermagem prescritas para pacientes adultos internados em unidade de terapia...I 6

Os dados obtidos pela extração manual das atividades de enfermagem prescritas foram transportados para o software Microsoft Office Excel 2013 e, após, submetidos à análise estatística descritiva com uso do Statistical Package for the Social Sciences (SPSS), versão 20. A análise dos dados foi feita por meio de estatística descritiva, em proporção percentual, e por meio da amplitude interquartil, sendo consideradas como atividades mais frequentes, as que apresentaram distribuição a partir do quartil 75\%.

A pesquisa foi desenvolvida de acordo com as normas da Resolução nº 466/2012 do Conselho Nacional de Saúde, recebendo parecer favorável de Comitê de Ética em Pesquisa (CEP) institucionalizado por meio de parecer $\mathrm{n}^{\mathrm{o}}$ : $1.025 .731 / 2015$.

\section{Resultados}

Foram avaliados 32 prontuários no recorte temporal estabelecido. Destes, 29 atenderam aos critérios de inclusão no estudo. Foram identificadas 725 atividades de enfermagem prescritas no momento da admissão do paciente crítico, as quais foram mapeadas de acordo com a classificação NIC.

A maioria dos prontuários $(n=16 ; 55,18 \%)$ era de pacientes do sexo masculino. A média de idade dos pacientes internados era de 51,27 anos, com variação de 18 a 75 anos, sendo que a maior parcela dos indivíduos internados $(\mathrm{n}=12 ; 41,37 \%)$ tinha mais de 60 anos de idade.

As 725 intervenções de enfermagem prescritas foram mapeadas de acordo com os sete domínios, com as 30 classes e com as 554 intervenções de enfermagem da classificação NIC. A Tabela 1 mostra a distribuição das intervenções prescritas, de acordo com as intervenções da referida classificação. 
7 | Stralhoti KNO, Matos FGOA, Alves DCI, Oliveira JLC, Berwanger DCI, Anchieta DW

Tabela 1 - Distribuição das atividades de enfermagem prescritas na admissão de pacientes na UTI, mapeadas de acordo com as intervenções de enfermagem da classificação NIC. Cascavel, 2015.

\begin{tabular}{ccc}
\hline Intervenções de Enfermagem Prescritas & n & $\mathbf{\%}$ \\
\hline Cuidados da pele: tratamentos tópicos & 113 & 15,59 \\
Controle da pressão & 85 & 11,73 \\
Supervisão & 72 & 9,93 \\
Controle de infecção & 63 & 8,69 \\
Precauções circulatórias & 47 & 6,49 \\
Cuidados com sondas e drenos & 45 & 6,20 \\
Monitoração neurológica & 32 & 4,41 \\
Posicionamento & 32 & 4,41 \\
Tratamento da febre & 29 & 4,00 \\
Tratamento da hipotermia & 29 & 4,00 \\
Manutenção da saúde oral & 28 & 3,86 \\
Aspiração de vias aéreas & 28 & 3,86 \\
Cuidado com os olhos & 27 & 3,73 \\
Manutenção de dispositivo para acesso venoso & 25 & 3,45 \\
Banho & 24 & 3,31 \\
Cuidados com os cabelos & 24 & 3,31 \\
Alimentação por sonda enteral & 16 & 2,20 \\
Cuidados com local da incisão & 6 & 0,83 \\
\hline Total & $\mathbf{7 2 5}$ & $\mathbf{1 0 0}$ \\
\hline
\end{tabular}

Segundo a amplitude interquartil, as intervenções de enfermagem que apresentaram frequência absoluta igual ou maior a 51 foram consideradas as intervenções mais frequentes por estarem contidas no quartil 75\%, sendo elas: “Cuidados da Pele: tratamentos tópicos” (n=113; 15,59\%), “Controle da Pressão" (n=85; 11,73\%), "Supervisão" (n=72; 9,93\%) e "Controle de Infecção” (n=63; 8,69\%).

A Tabela 2 evidencia a distribuição das intervenções atividades de enfermagem prescritas de acordo com as classes da classificação NIC. 
Tabela 2 - Distribuição das intervenções de enfermagem prescritas no momento da admissão de pacientes críticos na UTI, mapeadas de acordo com as classes da classificação NIC. Cascavel, 2015.

\begin{tabular}{ccc}
\hline $\begin{array}{c}\text { Classes da Classificação de Intervenções de } \\
\text { Enfermagem }\end{array}$ & $\mathbf{n}$ & $\mathbf{\%}$ \\
\hline Controle de Pele/Feridas & 204 & 28,15 \\
Facilitação do Autocuidado & 148 & 20,42 \\
Controle de Riscos & 135 & 18,62 \\
Controle da Perfusão Tissular & 72 & 9,93 \\
Termorregulação & 58 & 8,00 \\
Controle Neurológico & 32 & 4,41 \\
Controle de Imobilidade & 32 & 4,41 \\
Controle Respiratório & 28 & 3,86 \\
Suporte Nutricional & 16 & 2,20 \\
\hline Total & $\mathbf{7 2 5}$ & $\mathbf{1 0 0}$ \\
\hline
\end{tabular}

Os resultados da Tabela 2 mostram que das 30 classes contidas da classificação NIC, nove foram utilizadas pelos enfermeiros ao prescrever os cuidados de enfermagem no momento de admissão dos pacientes na UTI. Segundo a amplitude interquartil, as classes que apresentaram frequência absoluta igual ou maior a 141 foram consideradas as classes mais frequentes por estarem contidas no quartil 75\%, sendo elas: "Controle de Pele/Feridas” (n=204; 28,15\%) e “Facilitação do Autocuidado” (n=148; 20,42\%).

Por fim, a Tabela 3 ilustra as intervenções de enfermagem prescritas de acordo com os domínios da classificação NIC.

Tabela 3 - Distribuição das intervenções de enfermagem prescritas no momento da admissão de pacientes críticos na UTI, mapeadas de acordo com os domínios da classificação NIC. Cascavel, 2015.
Domínios da Classificação de Intervenções de
n $\%$
Enfermagem 
9 | Stralhoti KNO, Matos FGOA, Alves DCI, Oliveira JLC, Berwanger DCI, Anchieta DW

\begin{tabular}{ccc}
\hline Fisiológico Complexo & 394 & 54,34 \\
Fisiológico Básico & 196 & 27,03 \\
Segurança & 135 & 18,62 \\
Comportamental & - & - \\
Família & - & - \\
Sistema de Saúde & - & - \\
Comunidade & - & - \\
\hline Total & $\mathbf{7 2 5}$ & $\mathbf{1 0 0}$ \\
\hline
\end{tabular}

Com os resultados da Tabela 3, é possível visualizar que as intervenções de enfermagem prescritas para os pacientes críticos estavam compreendidas em três dos sete domínios existentes na classificação NIC. Segundo a amplitude interquartil, os domínios que apresentaram frequência absoluta igual ou maior a 196 foram considerados os domínios mais frequentes por estarem contidos no quartil 75\%, sendo eles: domínio "Fisiológico Complexo" (n=394; 54,34\%) e domínio “Fisiológico Básico” (n=196; 27,03\%).

\section{Discussão}

De acordo com os achados, das 554 possibilidades de intervenções de enfermagem classificadas na NIC, ${ }^{8} 18$ foram prescritas para os pacientes críticos no momento da admissão na UTI. As intervenções “Cuidados da Pele: tratamentos tópicos” (n=113; 15,59\%), “Controle da Pressão” (n=85; 11,73\%), “Supervisão” (n=72; 9,93\%) e o “Controle de Infecção" (n=63; 8,69\%) foram as intervenções mais frequentemente prescritas (Tabela 1).

Observando os resultados obtidos, percebe-se que de forma geral, os "Cuidados com a pele: tratamentos tópicos” e o "Controle da Pressão" obtiveram destaque. Esse fato pode ser atribuído ao déficit de mobilidade que os pacientes críticos possuem, devido à sua gravidade clínica e/ou fatores que impedem sua movimentação corporal. O perfil dos pacientes que necessitam de cuidados intensivos é de indivíduos acamados, com limitação ou dificuldade de 
Intervenções de enfermagem prescritas para pacientes adultos internados em unidade de terapia...। 10

movimentar-se no leito, o que demanda maiores cuidados para a manutenção da integridade da pele. $^{9}$

Importante destacar que a intervenção "Controle da pressão" não deixa de ser um “cuidado com a pele”, pois relaciona-se à perfusão tissular, logo, à prevenção e/ou tratamento de lesões de pele. Destarte, com base nos números elevados de lesões por pressão em pacientes que necessitam de cuidados intensivos, a enfermagem, junto à equipe multidisciplinar, deve planejar e executar suas ações com a finalidade de evitar o surgimento de lesões de pele. ${ }^{9}$

A intervenção de "Supervisão" também foi frequentemente prescrita. De acordo com a NIC, essa intervenção é definida como sendo: aquisição, interpretação e síntese contínuas, com a finalidade de obter dados do paciente para subsidiar a tomada de decisão clínica do enfermeiro. ${ }^{8}$ Essa intervenção engloba uma série de cuidados ligados ao monitoramento geral do paciente crítico, que exige supervisão permanente. Logo, interpreta-se que esta não é uma intervenção específica, mas que pode aumentar a segurança do doente criticamente enfermo pela supervisão atenta da equipe de enfermagem ao seu estado geral de saúde e sua evolução.

Cuidados como o controle da glicemia capilar estão inseridos na intervenção “supervisão" visto que paciente crítico pode apresentar elevação nos níveis glicêmicos, mesmo não sendo diabético. ${ }^{10}$ Outro cuidado relevante que está inserido na intervenção "supervisão" é o controle do padrão respiratório. Grande parcela dos pacientes internados na UTI encontra-se em ventilação mecânica $(\mathrm{VM})$ e nesses casos a observação do padrão respiratório é extremamente importante, pois inclui a avaliação de sinais e sintomas de possível infecção pulmonar, utilização de musculatura acessória, avaliação da ausculta pulmonar, controle do nível de saturação, entre outros. ${ }^{11}$

O “Controle de Infecção” também surge como uma intervenção de enfermagem frequentemente prescrita. Tendo em vista o elevado número de procedimentos invasivos realizados durante o período de internamento na UTI, o cuidado para evitar que o diagnóstico 
11 | Stralhoti KNO, Matos FGOA, Alves DCI, Oliveira JLC, Berwanger DCI, Anchieta DW

de risco de infecção se torne um diagnóstico real torna-se imprescindível. ${ }^{12}$ Para tanto, é necessário que a equipe de saúde reconheça os fatores que desencadeiam as Infecções Relacionadas à Assistência à Saúde (IRAS), bem como as medidas de prevenção cabíveis, de acordo com as melhores evidências. ${ }^{13}$

Como evidenciado na Tabela 2, as intervenções de enfermagem prescritas para os pacientes em estudo estavam inseridas em nove das 30 classes da classificação NIC ${ }^{8}$ sendo as mais frequentes: "Controle de Pele/Feridas" (n=204; 28,15\%) e "Facilitação do Autocuidado" $(n=148 ; 20,42 \%)$.

Os achados do presente estudo foram semelhantes aos obtidos em pesquisa realizada em UTI privada do Estado de Minais Gerais (Brasil), que identificou entre as classes mais frequentes o "Controle de Pele/Feridas", a "Facilitação do Autocuidado" e o "Controle de Riscos”. ${ }^{14}$ A alta frequência de tais classes pode ser justificada pelas particularidades dos pacientes críticos, pois são indivíduos com alta dependência assistencial, com necessidade de cuidados básicos e intensivos voltados à recuperação do seu estado de saúde, e que devido sua vulnerabilidade, estão sujeitos a várias situações que oferecem risco. ${ }^{15-16}$

A classe "Controle de Pele/Feridas" contempla as duas intervenções de enfermagem mais frequentemente prescritas: "Cuidados da Pele: tratamentos tópicos” e "Controle da pressão"; a classe “Controle de Riscos” abarca as outras duas intervenções mais frequentes: "Supervisão” e “Controle de Infecção”; já a classe "Facilitação do Autocuidado” contempla várias intervenções prescritas relacionadas a higiene corporal do paciente: "Manutenção da Saúde Oral”, "Cuidados com os Olhos”, “Banho” e "Cuidados com os Cabelos”.

A higiene corporal é fundamental para evitar o desenvolvimento de outros agravos à saúde, pois promove a remoção de células mortas e a redução da microbiota residente e transitória do paciente. ${ }^{17}$ Isso posto, cumpre ressaltar a baixa proporção de intervenções prescritas a respeito de: banho, cuidados com os olhos, e cuidados com os cabelos, que, apesar 
Intervenções de enfermagem prescritas para pacientes adultos internados em unidade de terapia...। 12

de possivelmente perfazerem cuidados de cunho rotineiro, merecem prescrição pelo enfermeiro a fim de reforçar sua relevância.

Mesmo não estando contida no quartil 75\%, a prescrição de atividades da classe "Controle de riscos" demonstra a importância atribuída aos cuidados que promovam a segurança do paciente. A atenção das instituições sobre esse assunto acontece no âmbito mundial visto que a assistência ao paciente é prestada por seres humanos que, por sua vez, são passíveis de erros. ${ }^{18}$ Devido a sua posição estratégica na gestão do cuidado, o enfermeiro é um ator comumente requerido na implantação e monitoramento de ações voltadas à segurança do paciente. ${ }^{19}$

Como evidenciado na Tabela 3 , as atividades de enfermagem prescritas para os pacientes críticos do hospital em estudo estavam inseridas em três dos sete domínios da classificação NIC, sendo que os mais frequentes foram: "Fisiológico Complexo" (n=394; 54,34\%) e "Fisiológico Básico” (n=196; 27,03\%).

Os resultados da presente pesquisa corroboram com achados anteriores, também no contexto brasileiro, em que o maior número de atividades prescritas para pacientes críticos estava contida nos domínios “Fisiológico Complexo”, "Fisiológico Básico” e "Segurança”. ${ }^{18}$ A interpretação de tais achados viabiliza a compreensão de que os cuidados de enfermagem em UTI visam a manutenção da vida do doente gravemente enfermo.

Importante destacar que o paciente crítico apresenta déficit no autocuidado, justificando a alta frequência da prescrição de atividades contidas no domínio "Fisiológico Básico". 8 O cerne da enfermagem é o cuidado humano, que em suas bases da profissão, consiste na evidência de que a adequada higiene corporal e do ambiente auxilia no restabelecimento da saúde. ${ }^{20}$

Apesar da prescrição de ações de enfermagem contidas nos demais domínios da classificação NIC serem comumente menos frequentes, acredita-se que as mesmas tenham 
13 | Stralhoti KNO, Matos FGOA, Alves DCI, Oliveira JLC, Berwanger DCI, Anchieta DW

adequada aplicabilidade aos pacientes críticos, visto que os mesmos possuem uma "família”, estão inseridos em uma determinada “comunidade” e fazem parte de um "sistema de saúde”.

O baixo número de prescrições contidas nos domínios de focos psicossociais pode estar relacionado às condições inadequadas de trabalho, visto que é comum encontrar unidades assistenciais comportando número excessivo de pacientes, equipe de funcionários reduzida, rotinas com elevada carga horária e volume excessivo de trabalho. ${ }^{21}$ Nesse contexto, talvez os enfermeiros acabem priorizando as prescrições voltadas ao cuidado biológico. No entanto, não valorizar cuidados de enfermagem que vão além das ações curativas pode fragilizar a ascensão da profissão, e possivelmente, contribuir para a manutenção do modelo assistencial biomédico.

\section{Conclusão}

Conclui-se que as intervenções de enfermagem mais frequentes para o paciente crítico foram voltadas à manutenção da vida, por meio de ações que entornam o controle fisiológico. Ainda, ações de segurança também emergiram por um concentrado de intervenções de enfermagem.

O estudo aponta que, pela instabilidade clínica dos pacientes em estado crítico, os enfermeiros intensivistas possivelmente priorizam a prescrição de cuidados voltados para atender as disfunções fisiológicas em detrimento dos cuidados que atendam as necessidades comportamentais, familiares, psicossociais e espirituais. Outro ponto a destacar é a baixa proporção de intervenções relacionadas ao cuidado corporal.

A amostra reduzida e a impossibilidade de inferir os resultados para outras realidades são limitações expressas à pesquisa. Todavia, acredita-se que o estudo traz contribuições à prática sistematizada da enfermagem, em especial por divulgar o uso de classificação internacional no contexto do cuidado intensivo, o que fortalece o trabalho de gestão do cuidado do enfermeiro em UTI e também, coaduna à consolidação da enfermagem como ciência. 


\section{Referências}

1. Soares MI, Resck ZMR, Terra FS, Camelo SHH. Sistematização da assistência de enfermagem: facilidades e desafios do enfermeiro na gerência da assistência. Esc Anna Nery Rev Enferm [Internet]. 2015 jan-mar [acesso em 2018 jun 29];19(1):47-53. Disponível em: http://www.scielo.br/pdf/ean/v19n1/1414-8145-ean-19-01-0047.pdf doi: http://dx.doi.org/10.5935/14148145.20150007

2. Conselho Regional de Enfermagem (COREN-SP). Processo de enfermagem: guia para a prática. São Paulo (SP): COREN-SP; 2015.

3. Conselho Federal de Enfermagem (COFEN). Resolução n. 358, de 15 de outubro de 2009. Dispõe sobre a sistematização da assistência de enfermagem e a implementação do processo de enfermagem em ambientes, públicos ou privados, em que ocorre o cuidado profissional de enfermagem, e dá outras providências [Internet]. 2009 [acesso em 2018 jul 02]. Disponível em: http://www.portalcofen.gov.br/sitenovo/node/4384

4. Gutiérrez MGR, Morais SCRV. Sistematização da Assistência de Enfermagem e a

formação da identidade profissional. Rev Bras Enferm [Internet]. 2017 mar-abr [acesso em 2018 jun 29];70(2):436-41. Disponível em: http://www.scielo.br/scielo.php?script=sci_arttext\&pid=S003471672017000200436\&lng=en\&tlng=en doi: http://dx.doi.org/10.1590/0034-7167-2016-0515

5. Lesmes AG, Torres CA, Rodríguez NJL. Factores relacionados con la aplicación del proceso de enfermería en instituciones hospitalarias de Colombia. Cuid [Internet]. 2016 [acesso em 2018 jun 29];9(1):2007-16. Disponível em: https://www.revistacuidarte.org/index.php/cuidarte/article/view/480/917 doi: https://doi.org/10.15649/cuidarte.v9i1.480

6. Fiorin JMA, Schran LS, Oliveira JLC, Maraschin MS, Alves DCI, Tonini NS. Avaliação da qualidade de prescrição de enfermagem em unidade de terapia intensiva. Enferm Atual [Internet]. 2018 [acesso em 2018 jul 03];85(23):29-36. Disponível em: https://revistaenfermagematual.com.br/revista/23/avaliacao-daqualidade-de-prescricoes-de-enfermagem-em-unidade-de-terapia-intensiva doi: http://dx.doi.org/10.1590/S1414-81452012000300021

7. Sanches RCN, Gerhardt PC, Rêgo AS, Carreira L, Pupulim JSL, Radovanovic CAT. Percepções de profissionais de saúde sobre a humanização em unidade de terapia intensiva adulto. Esc Anna Nery Rev Enferm [Internet]. 2016 jan-mar [acesso em 2018 jun 29];20(1): 48-54. Disponível em: http://www.scielo.br/pdf/ean/v20n1/1414-8145-ean-20-01-0048.pdf doi: http://dx.doi.org/10.5935/14148145.20160007

8. Bulechek GM, Butcher HK, Dochterman JM, Wagner CM. Classificação das intervenções de enfermagem (NIC). 6ª ed. Rio de Janeiro (RJ): Elsevier; 2016. 
9. Constantin AG, Moreira APP, Oliveira JLC, Hofstätter LM, Fernandes LM. Incidence of pressure injury in an adult intensive care unit. Rev Estima [Internet]. 2018 [acesso em 2018 jun 29];16:e1118. Disponível em: https://www.revistaestima.com.br/index.php/estima/article/view/454/pdf doi: http://dx.doi.org/10.30886/estima.v16.454

10. Silva WO. Controle glicêmico em pacientes críticos na UTI. Rev Hosp Univ Pedro Ernesto [Internet]. 2013 [acesso em 2018 jun 29];12(3):47-56. Disponível em: http://www.epublicacoes.uerj.br/index.php/revistahupe/article/view/7530 doi: http://dx.doi.org/10.12957/rhupe.2013.7530

11. Truppel TC, Meier MJ, Calixto RC, Peruzzo AS, Crozeta K. Sistematização da assistência de enfermagem em Unidade de Terapia Intensiva. Rev Bras Enferm [Internet]. 2009 mar-abr [acesso em 2018 jun 29];62(2):221-7. Disponível em: http://www.scielo.br/scielo.php?script=sci_arttext\&pid=S003471672009000200008\&lng=pt\&tlng=pt doi: http://dx.doi.org/10.1590/S0034-71672009000200008

12. Guedes DMB, Santos LC, Oliveira EA. Intervenções de enfermagem em uma unidade de terapia intensiva pediátrica. Rev Enferm UFPE On Line [Internet]. 2017 jan [acesso em 2018 jul 04];11(1):102-11. Disponível em: https://periodicos.ufpe.br/revistas/revistaenfermagem/article/view/11883 doi: 10.5205/reuol.9978-88449-6-1101201713

13. Lorenzini E, Costa TC, Silva EF. Prevenção e controle de infecção em unidade de terapia intensiva neonatal. Rev Gaúch Enferm [Internet]. 2013 dez [acesso em 2018 jun 29];34(4):107-13. Disponível em: http://www.scielo.br/scielo.php?script=sci_arttext\&pid=S1983-14472013000400014\&lng=pt\&tlng=pt doi: http://dx.doi.org/10.1590/S1983-14472013000400014

14. Salgado PO, Chianca TCM. Identificação e mapeamento dos diagnósticos e ações de enfermagem em unidade de terapia intensiva. Rev Latinoam Enferm [Internet]. 2011 jul-ago [acesso em 2018 jun 29];19(4):928-35. Disponível em: http://www.scielo.br/pdf/rlae/v19n4/pt_11.pdf doi: http://dx.doi.org/10.1590/S0104-11692011000400011

15. Ortega DB, D'innocenzo M, Silva LMG, Bohomol E. Análise de eventos adversos em pacientes internados em unidade de terapia intensiva. Acta Paul Enferm [Internet]. 2017 mar-abr [acesso em 2018 jun 29];30(2):168-73. Disponível em: http://www.scielo.br/scielo.php?script=sci_arttext\&pid=S010321002017000200168\&lng=pt\&tlng=pt doi: http://dx.doi.org/10.1590/1982-0194201700026

16. Roque KE, Tonini T, Melo EC. Adverse events in the intensive care unit: impact on mortality and length of stay in a prospective study. Cad Saúde Pública [Internet] 2016 out [acesso em 2018 jun 29];32(10):e00081815. Disponível em: http://www.scielo.br/scielo.php?script=sci_arttext\&pid=S0102311X2016001005001\&lng=e doi: http://dx.doi.org/10.1590/0102-311X00081815

17. Benedet SA, Brasil N. A sistematização da assistência de enfermagem e as necessidades de cuidados de pacientes internados em terapia intensiva. Rev Eletrônica Gest Saúde [Internet] 2012 [acesso em 2018 jun 29];3(2):522-37. Disponível em: https://dialnet.unirioja.es/descarga/articulo/5555774.pdf 
18. Salgado PO, Tannure MC, Oliveira CR, Chianca TCM. Identificação e mapeamento das ações de enfermagem prescritas para pacientes internados em uma UTI de adultos. Rev Bras Enferm [Internet]. 2012 mar-abr [acesso em 2018 jun 29];65(2):291-6. Disponível em: http://www.scielo.br/scielo.php?script=sci_arttext\&pid=S0034-71672012000200014\&lng=pt\&tlng=pt doi: http://dx.doi.org/10.1590/S0034-71672012000200014

19. Oliveira JLC, Reis GAX, Souza VS, Costa MAR, Valera IMA, Matsuda LM. Facilitating factors in the implementation of patient safety strategies: a descriptive exploratory study. Online Braz j Nurs [Internet]. 2017 jun [acesso em 2018 jul 02];16(2):108-18. Disponível em: http://www.objnursing.uff.br/index.php/nursing/article/view/5515 doi: http://dx.doi.org/10.17665/16764285.20175515

20. Costa GS, Souza CC, Diaz FBBS, Toledo LV, Ercole FF. Banho no leito em cuidados críticos: uma revisão integrativa. Rev Baiana Enferm [Internet]. 2018 [acesso em 2018 jul 04];32:e20483. Disponível em: https://portalseer.ufba.br/index.php/enfermagem/article/view/20483 doi: http://dx.doi.org/10.18471/rbe.v32.20483

21. Borges F, Bohrer CD, Bugs TV, Nicola AL, Tonini NS, Oliveira JLC. Dimensionamento de pessoal de enfermagem na UTI-Adulto de hospital universitário público. Cogitare Enferm [Internet]. 2017 [acesso em 2018 jun 29];2(22):e50306. Disponível em: https://revistas.ufpr.br/cogitare/article/view/50306 doi: http://dx.doi.org/10.5380/ce.v22i2.50306

\section{Autor correspondente}

Fabiana Matos

E-mail: fabianamatos@hotmail.com

Endereço: Rua Monjoleiro, $\mathrm{n}^{\mathbf{0}}:$ 125, Tropical, Cascavel-PR

CEP: $85807-300$

\section{Contribuições de Autoria}

1 - Kalliny Nathiara de Oliveira Stralhoti

Contribuições substanciais na concepção ou desenho do trabalho, bem como na coleta, análise e interpretação dos dados. Participação na redação do artigo e na sua revisão crítica. Na aprovação final da versão a ser publicada.

2 - Fabiana Gonçalves de Oliveira Azevedo Matos

Contribuições substanciais na concepção ou desenho do trabalho, bem como na coleta, análise e interpretação dos dados. Participação na redação do artigo e na sua revisão crítica. Na aprovação final da versão a ser publicada.

3 - Débora Cristina Ignácio Alves

Participação na redação do artigo e na sua revisão crítica. Na aprovação final da versão a ser publicada. 
17 | Stralhoti KNO, Matos FGOA, Alves DCI, Oliveira JLC, Berwanger DCI, Anchieta DW

4 - João Lucas Campos de Oliveira

Participação na redação do artigo e na sua revisão crítica. Na aprovação final da versão a ser publicada.

5 - Djulia Camila Berwanger

Participação na redação do artigo e na sua revisão crítica. Na aprovação final da versão a ser publicada.

6 - Drieli Wawzeniak de Anchieta

Participação na redação do artigo e na sua revisão crítica. Na aprovação final da versão a ser publicada.

\section{Como citar este artigo}

Stralhoti KNO, Matos FGOA, Alves DCI, Oliveira JLC, Berwanger DCI, Anchieta DW. Intervenções de enfermagem prescritas para pacientes adultos internados em unidade de terapia intensiva. Rev. Enferm. UFSM. 2019 [Acesso em: Anos Mês Dia];vol e2: P1-P16. DOI:https://doi.org/10.5902/2179769233373 